\title{
Heritability estimates on Hodgkin's lymphoma: a genomic- versus population-based approach
}

\author{
Hauke Thomsen ${ }^{\star, 1}$, Miguel Inacio da Silva Filho ${ }^{1}$, Asta Försti ${ }^{1,2}$, Michael Fuchs ${ }^{3}$, Sabine Ponader ${ }^{3}$, \\ Elke Pogge von Strandmann ${ }^{3}$, Lewin Eisele ${ }^{4}$, Stefan Herms ${ }^{5,6}$, Per Hofmann ${ }^{5,6}$, Jan Sundquist ${ }^{7}$, \\ Andreas Engert ${ }^{3}$ and Kari Hemminki ${ }^{1,2}$
}

Genome-wide association studies (GWASs) have identified several single-nucleotide polymorphisms (SNPs) influencing the risk of Hodgkin's lymphoma (HL) and demonstrated the association of common genetic variation for this type of cancer. Such evidence for inherited genetic risk is also provided by the family history and the very high concordance between monozygotic twins. However, little is known about the genetic and environmental contributions. A common measure for describing the phenotypic variation due to genetics is the heritability. Using GWAS data on $906 \mathrm{HL}$ cases by considering all typed SNPs simultaneously, we have calculated that the common variance explained by SNPs accounts for $>35 \%$ of the total variation on the liability scale in $\mathrm{HL}(95 \%$ confidence interval $6-62 \%)$. These findings are consistent with similar heritability estimates of $\sim 0.40(95 \%$ confidence interval 0.17-0.58) based on Swedish population data. Our estimates support the underlying polygenic basis for susceptibility to $\mathrm{HL}$, and show that heritability based on the population data is somehow larger than heritability based on the genomic data because of the possibility of some missing heritability in the GWAS data. Besides that there is still major evidence for multiple loci causing $\mathrm{HL}$ on chromosomes other than chromosome 6 that need to be detected. Because of limited findings in prior GWASs, it seems worth checking for more loci causing susceptibility to HL.

European Journal of Human Genetics (2015) 23, 824-830; doi:10.1038/ejhg.2014.184; published online 17 September 2014

\section{INTRODUCTION}

Hodgkin's lymphoma (HL) is a malignancy of the lymphatic system with an incidence of 2-3/100 000/year in developed countries. ${ }^{1}$ It is characterized through malignant Hodgkin and Reed-Sternberg (HRS) cells mixed with a dominant background population of reactive lymphocytes and other inflammatory cells. ${ }^{2}$ Epstein-Barr virus (EBV) infections may be causally related to a number of cases as well as a personal history of autoimmune diseases. ${ }^{3,4}$ However, there is limited evidence to the involvement of other specific environmental risk factors, although there is a distinctive pattern of incidence rates and risk profiles by age, race/ethnicity, sex and economic levels. ${ }^{2}$ Some evidence for inherited genetic influence on susceptibility is provided by the increased familial risk and the very high concordance between monozygotic twins. ${ }^{5}$ Recently, several genome-wide association studies (GWASs) have identified a couple of loci for HL. ${ }^{6,7}$ These studies have shown that the risk of HL is highly influenced by the human leukocyte antigen (HLA) genotype variation, but the familial risk is also a consequence of non-HLA genotype variation. ${ }^{6}$ However, all the genetic variants identified so far only capture a minor percentage of the estimated heritability of the disease. ${ }^{8}$ Yet, a great deal remains to be understood regarding the remaining heritability. ${ }^{9,10}$ Some plausible explanation include unidentified gene-gene interactions, unidentified contributions of rare genetic variants or overestimating the total heritability for $\mathrm{HL}$ in population-based studies, resulting in a 'phantom heritability', 9,11,12 that cannot be dissolved on the molecular basis. ${ }^{13,14}$

Thus, our aim is to provide reliable estimates for the genetic variation of the disease derived either from the quantification of resemblance between close relatives or the dissection of genetic variation from genomic loci. ${ }^{15}$

The knowledge of the heritability estimates for the susceptibility to HL based on genomic data and on population data will then provide further insights in the proportion of genetic variation hidden so far but still detectable on the genomic level. ${ }^{16}$

\section{MATERIALS AND METHODS}

Genomic data: quality control of SNP genotyping

The study population comprised a total of 2227 individuals, with 1001 cases and 1226 controls. Cases were sampled all over Germany, whereas controls were sampled within the Ruhr area in North Rhine-Westphalia as part of the Heinz Nixdorf Recall Study. ${ }^{17}$ All individuals were genotyped using the Illumina Human Omni Express 12v1 chip (Illumina, San Diego, CA, USA). (733 202 markers).

To counteract artificial differences in allele frequencies between cases and controls causing spurious genetic variation, GWAS data have undergone a very stringent quality control procedure. ${ }^{16}$ After checking the gender based on genotypes, 11 individuals were excluded because of inconsistencies. Three individuals were excluded because of low genotype calling rates $(<0.99)$. In total, 27 individuals were excluded, because their heterozygosity was $>3 \mathrm{SD}$ apart from the mean heterozygosity of the sample. Principal component

${ }^{1}$ German Cancer Research Center (DKFZ), Division of Molecular Genetic Epidemiology, Heidelberg, Germany; ${ }^{2}$ Center for Primary Health Care Research, Lund University, Malmö, Sweden; ${ }^{3}$ Department of Internal Medicine I, University Hospital of Cologne, Cologne, Germany; ${ }^{4}$ Institute for Medical Informatics, Biometry and Epidemiology, University Hospital Essen, University Duisburg-Essen, Essen, Germany; ${ }^{5}$ Institute of Human Genetics and Department of Genomics, University of Bonn, Bonn, Germany; ${ }^{6}$ Division of Medical Genetics, Department of Biomedicine, University of Basel, Basel, Switzerland; ${ }^{7}$ Stanford Prevention Research Center, Stanford University School of Medicine, Stanford, CA, USA *Correspondence: Dr H Thomsen, German Cancer Research Center (DKFZ), Division of Molecular Genetic Epidemiology, C050, Im Neuenheimer Feld 580, 69120 Heidelberg, Germany. Tel: +49 6221 421809; Fax: +49 6221 421810; E-mail: h.thomsen@dkfz-heidelberg.de

Received 31 December 2013; revised 6 August 2014; accepted 10 August 2014; published online 17 September 2014 
analysis indicated a presence of population outliers, especially in the cases, because cases represent a more diverse group than controls. After excluding these 46 outliers, the remaining individuals were genetically well matched. Seventeen individuals having a relatedness score of $>0.05$ were excluded. The final set consisted of 906 cases and 1217 controls. All data were checked for differential missingness between cases and controls, and single-nucleotide polymorphisms (SNPs) were excluded with $P<0.05$. SNPs were also excluded after applying the Hardy-Weinberg equilibrium test with $P<0.001$. A detailed overview of the study material, the identification of samples of non-European origin, the plots of the principal components and the results is given in our previous paper. $^{6}$ The genome-wide Armitage trend test $\chi 2$ values showed minimal inflation of the test statistics proving the absence of substantial cryptic population substructure (genomic control inflation factor $\lambda_{\mathrm{gc}}=1.09$ ). ${ }^{6}$

By using PLINK software ${ }^{18}$ we finally produced two subsets of data with SNPs in cases and controls that had either a minor allele frequency (MAF) of $>0.01$ or MAF of $>0.05$.

\section{Statistical analysis on genomic data}

For the statistical analyses on the genomic data, the approach of Yang et al ${ }^{19}$ was used. Their method has been completed by an approach of Speed et al, ${ }^{20}$ who presented an improved method for the heritability estimation on GWAS data with a new adjustment for linkage disequilibrium (LD). Both methods provide an estimate of the additive genetic variance explained by SNPs but are accounting for LD between the genotyped SNPs and unknown causal variants in different ways (ie, correlations between SNP genotypes). ${ }^{19,20}$ Both approaches fit a linear mixed model of the form: $y=\mu+g+e,{ }^{16,19}$ whereby $y$ is the vector of the disease status, $\mu$ is the mean vector, $\mathrm{g}$ is a vector of random additive genetic effects obtained from SNP data and e is a vector of residual effects. The covariance structure fitted in the data is the individual relationship estimated from the SNPs; $\operatorname{cov}\left(y_{j}, y_{k}\right)=A_{j k} \sigma_{g}^{2}+\sigma_{e}^{2}$, where $A_{j k}$ is the genetic relationship between individuals $j$ and $k$ derived from the SNPs, $\sigma_{g}^{2}$ is the additive genetic variance and $\sigma_{e}^{2}$ is the residual variance. With this model disease heritability, $h_{0}^{2}$, can be defined as: $\sigma_{g}^{2} /\left(\sigma_{g}^{2}+\sigma_{e}^{2}\right){ }^{20}$ Because phenotypes in case-control studies are measured on the $0-1$ scale, the relationship between observations on the observed scale and liabilities on the unobserved continuous scale are modeled through the liability threshold model. The liability for HL on the underlying scale follows a standard normal distribution whereby if liability exceeds a certain threshold, $t$, then individuals will be affected. The estimate of variance explained by the SNPs on the observed $0-1$ scale is linearly transformed to that on the unobserved continuous liability scale such that $h_{l}^{2}=h_{0}^{2} K(1-K) / z^{2},{ }^{21}$ where $K$ is the prevalence of the disease and $z$ is the value of the standard normal probability density function at the threshold $t$. Given an incidence of 2-3/100 000/year will result in a cumulative risk of $\sim 1$ in 1000, which can be considered as an estimate of the prevalence. The relationship between additive genetic variance on the observed $0-1$ and unobserved liability scales is extended to account for ascertainment bias in a case-control study. ${ }^{16}$ Estimation of the additive genetic variance was performed using restricted maximum likelihood (REML) via the genome-wide complex trait analysis (GCTA) software. ${ }^{22}$ Because the MAF spectrum of the unobserved causal variants may be different from the genotyped SNPs, the estimation of the variance explained by SNPs was performed in two ways. (1) the estimate of the variance explained by SNPs was adjusted to account for missing LD between the genotyped SNPs and unknown causal variants. ${ }^{19}$ SNPs were randomly assigned into two different groups with one of the groups being treated as representing 'true' causal variants. The covariance between both groups is supposed to reflect the true variance of relatedness between individuals, whereas the variance derived from the SNP group equals the variation of relatedness plus estimation error. The prediction error can then be derived by regressing the relationships of the 'true' causal variants on the SNPs. (2) In contrast to the method of Yang et al, ${ }^{19}$ which suggests a uniformly scaling of the usual SNP-based kinship coefficients, Speed et al ${ }^{20}$ proposed a different adjustment, in which SNPs are weighted according to how well they are tagged by their neighbors. The kinship coefficients corrected for $\mathrm{LD}$ are obtained in a two-step procedure: first, weightings for each predictor given the local patterns of correlations are calculated, and second these weightings are used to estimate relatedness values across all pairs of individuals. ${ }^{20}$ The final estimation of the additive genetic variance was again performed by using REML via the software tool GCTA.22

In addition, the approach of Guan and Stephen ${ }^{23}$ has been applied to estimate the proportion of phenotypic variance explained (PVE) by the genotypes. It implements the Markov chain Monte Carlo (MCMC)-based inference methods for Bayesian variable-selection regression based on standard normal linear regression with the following model:

$$
\mathrm{y} \mid \mu, \beta, X, \tau \sim N n\left(\mu+X \beta, \tau^{-1} \mathrm{I}_{n}\right),
$$

relating a response variable y to covariates $X$. Here $\mathrm{y}$ is an $n$-vector of observations on $n$ individuals, $\mu$ is an $n$-vector with components all equal to the same scalar $\mu, X$ is an $n$ by $p$ matrix of covariates, $\beta$ is a $p$-vector of regression coefficients, $\tau$ denotes the inverse variance of the residual errors, $N n(\cdot, \cdot)$ denotes the $n$-dimensional multivariate normal distribution and $\mathrm{I}_{n}$ the $n$ by $n$ identity matrix. The variables $\mathrm{y}$ and $X$ are observed, whereas $\mu, \beta$ and $\tau$ are parameters to be inferred. Because GWAS applications involve binary phenotypes, the probit link function is used and allows direct comparisons to the estimates of variance explained by SNPs on the liability scale. The total proportion of variance in y explained by the relevant covariates $X_{\gamma}$, or PVE, is commonly used to summarize the results of a linear regression.

The PVE is closely related to the 'heritability' of the phenotype and reflects the optimal predictive accuracy that could be achieved for a linear combination of the measured genetic variants, whereas heritability reflects the accuracy that could be achieved by all genetic variants. ${ }^{23}$

\section{Population data: Swedish Family-Cancer Database}

To compare the variance explained by SNPs to new estimates of heritability from family-based studies, variance components were estimated for the susceptibility to HL on the basis of the 2010 update of the Swedish FamilyCancer Database that includes all individuals born after 1931 who are residing in Sweden, together with their biological parents, totaling $\sim 12.1$ million individuals. ${ }^{24}$ The Database was created in 1996 by combining the Swedish Cancer Registry and the Swedish Multigenerational Register, and has been updated regularly. The Database includes information about the cancers, socioeconomic data and death causes. In total, 7438 individuals (4441 males and 2997 females) have been diagnosed with the HL (ICD-7 code 201). The R-stat package and DmuTrace ${ }^{25,26}$ were used to extract all related individuals of the patients from the large pedigree file back to the base population as well as all offspring of the patients and their relatives in future generations until the current population. This resulted in a pedigree of 133544 individuals (67 059 males and 66485 females). The entire pedigree consisted of 6755 families across 6 generations with a family size ranging from 2 to 490 individuals. The total number of founders was 59679 with a range of 2 to 203 individuals per family. Each family contained at least one and up to eight cases.

\section{Statistical analysis on population data}

A generalized linear mixed effect threshold model with a binary response variable using MCMC Carlo techniques was applied. ${ }^{27}$ In such a standard threshold (probit) model, the observed binary records $\left(Y_{i j}\right)$ are assumed fully determined by an underlying liability $\left(\lambda_{i t}\right)$, such that: for $Y_{i j}=0$ for $\lambda_{i j} \leq 0$ and $Y_{i j}=1$ for $\lambda_{i j}>0$ and the threshold value is set to 0 . The model can be written as

$$
\lambda=X \beta+\mathrm{Za}+e
$$

where $\lambda=$ vector of all $\lambda_{i j}$, $\beta=$ vector of 'fixed' effects, $\mathrm{a}=$ vector of random additive genetic effects of all individuals, $\mathrm{e}=$ vector of random residuals and $\mathrm{X}$ and $\mathrm{Z}$ are the appropriate incidence matrices. $\operatorname{Var}(\mathrm{a})=\mathrm{A} \sigma_{\mathrm{a}}^{2}$ and $\operatorname{Var}(\mathrm{e})=\mathrm{I}_{\mathrm{n}} \sigma_{\mathrm{e}}{ }_{\mathrm{e}}$, where $A$ is the additive genetic relationship matrix of all individuals, $I_{n}$ is an identity matrix with dimension equal to number of records and $\sigma_{\mathrm{a}}{ }^{2}$ and $\sigma_{\mathrm{e}}{ }^{2}$ are the additive genetic and the residual variances, respectively. As usual for probit threshold models, $\sigma_{\mathrm{e}}^{2}$ is restricted to be 1. Fixed effects included in the model were gender, birth year, country of birth, social economic index and number of children.

Marginal estimates of the genetic parameters were obtained in the underlying scale using Bayesian inference, implemented via the Gibbs sampling procedure and a data augmentation approach. The model included a Gibbs sampling chain of 10150000 rounds with a conservative 150000 iterations as burn-in 
and 10 million sampling rounds. Every $1000^{\text {th }}$ sample was drawn, resulting in 10000 samples. For each sample of the Gibbs chain, narrow-sense heritability was calculated after as: $\mathrm{h}^{2}=\sigma_{\mathrm{a}}^{2} /\left(\sigma_{\mathrm{a}}^{2}+\sigma_{\mathrm{e}}^{2}\right)$, where $\sigma_{\mathrm{e}}^{2}=1$ for probit link model. ${ }^{28,29}$

Posterior marginal means of heritability were derived and the $95 \%$ highest marginal posterior density region of the heritability range determined. The algorithm to estimate genetic (co)variance components has been implemented in the Gibbs sampling module of the DMU statistical software package ${ }^{30}$ that has been developed to handle multivariate genetic analyses including binary, ordered categorical and Gaussian traits. Results have been proven by the $\mathrm{R}$ package MCMCglmm ${ }^{31}$ that has also been used to prove convergence diagnosis and output analysis. Heritability estimates on the liability scale have been transformed to the observed scale by using the linear transformation of Dempster and Lerner. ${ }^{21}$

\section{RESULTS}

\section{Estimates of the variance explained by SNPs}

The analysis on genomic data was restricted to the autosomes of the GWAS data set and based on the final set of 906 cases and 1217 controls. For both primary subsets of genomic data with either MAF $>0.05$ or MAF $>0.01$, the threshold for SNP missingness was raised stepwise starting from 0.05 up to 0.001 . This resulted in a reduced number of SNPs from 583333 to 442325 (MAF >0.01). Both the crude proportions of variance and the adjusted estimates only dropped slightly, whereas the transformed estimate was stable across all subsets with different numbers of SNPs (Table 1). We only included adjusted estimates according to Yang et al ${ }^{19}$ in our tables as they did not show any difference to the adjustment according to Speed et al. ${ }^{20}$ Standard errors were slightly larger for the adjusted estimates and the transformed estimates. In contrast to stable estimates across different numbers of SNPs, the PVE showed moderate differences when using the Bayesian variable selection approach (Table 2). For data sets with less SNPs, the PVE values decrease and the high posterior density interval is shrinking. This fact is also presented in Figures 1 and 2 that show the posterior distributions of PVE from the MCMC runs for the corresponding MAF and each SNP subset. Values for PVE were larger when compared with the transformed estimate in Table 1.

Although the genomic control inflation factor was still small and mainly influenced by the distribution of cases collected across Germany, ${ }^{6}$ genomic partitioning was used to check the final influence of the population structure. ${ }^{20,32}$ Estimates were derived for chromosomes $1-7$ and for the remaining chromosomes (8-22). For any threshold shown in Table 1, estimates of the two parts of the genome added up to the total estimates of common variance explained by SNPs as described in Table 1 . The influence of the population structure has then also been tested with an additional REML analysis by fitting the first 2, 4 and 10 eigenvectors from the PCA as covariates. The results in Table 3 show little to no difference in the crude estimates of the variance explained by SNPs compared with original results in Table 1.

As many SNPs on chromosome 6 had extremely significant associations, one analysis was performed by excluding chromosome 6 , or by analyzing chromosome 6 separately just as in the genome partitioning approach. ${ }^{20}$ The results in Table 4 show that estimates (crude and adjusted) decreased by $\sim 15-20 \%$ when excluding chromosome 6 (as compared with Table 1), whereas estimates based on SNPs on chromosome 6 solely account for $\sim 5 \%$ of the variance explained by SNPs. This analysis shows that a substantial proportion of genetic variation is explained by risk loci on chromosome 6, yet another big proportion of the genetic variation is still explained by SNPs on other chromosomes.

Even though the incidence of 2-3/100 000/year has been stable for a long time, ${ }^{2}$ any variation over time because of changes in the

\section{Table 2 Proportion of estimated variance (PVE)}

\begin{tabular}{|c|c|c|c|c|}
\hline Threshold & No. of SNPS & Interations sampled & PVE means ${ }^{\mathrm{a}}$ & $\begin{array}{l}\text { Highest posterior } \\
\text { density }(\mathrm{Cl}=0.95)^{\mathrm{b}}\end{array}$ \\
\hline \multicolumn{5}{|l|}{$M A F^{\top}>0.01$} \\
\hline GENO $^{d}>0.05$ & 583333 & 10000 & 0.45 & $0.13-0.62$ \\
\hline GENO > 0.01 & 579445 & 10000 & 0.44 & $0.16-0.61$ \\
\hline GENO >0.005 & 566831 & 10000 & 0.39 & $0.17-0.53$ \\
\hline GENO $>0.001$ & 442325 & 10000 & 0.42 & $0.19-0.55$ \\
\hline \multicolumn{5}{|l|}{$M A F>0.05$} \\
\hline GENO >0.05 & 540228 & 10000 & 0.43 & $0.06-0.60$ \\
\hline GENO >0.01 & 536668 & 10000 & 0.48 & $0.09-0.62$ \\
\hline GENO $>0.005$ & 525248 & 10000 & 0.40 & $0.07-0.57$ \\
\hline GENO >0.001 & 410973 & 10000 & 0.39 & $0.11-0.54$ \\
\hline
\end{tabular}

${ }^{a}$ Means of PVE.

b95\% Confidence interval of the highest posterior density.

cMinor allele frequency.

${ }^{d}$ Maximum per-SNP missing rate.

Table 1 Estimated genetic variances for different setups

\begin{tabular}{|c|c|c|c|c|}
\hline Threshold & No. of SNPS & Estimate $(S E)^{a}$ & Adjusted $(S E)^{b}$ & Transformed (SE) \\
\hline \multicolumn{5}{|l|}{$M A F^{\mathrm{d}}>0.01$} \\
\hline GENO $^{\mathrm{e}}>0.05$ & 583333 & $0.24(0.03)$ & $0.24(0.05)$ & $0.35(0.06)$ \\
\hline GENO >0.01 & 579445 & $0.23(0.03)$ & $0.23(0.05)$ & $0.35(0.06)$ \\
\hline GENO >0.005 & 566831 & $0.23(0.03)$ & $0.23(0.05)$ & $0.35(0.06)$ \\
\hline GENO > 0.001 & 442325 & $0.22(0.03)$ & $0.22(0.05)$ & $0.35(0.05)$ \\
\hline \multicolumn{5}{|l|}{$M A F>0.05$} \\
\hline GENO >0.05 & 540228 & $0.23(0.03)$ & $0.23(0.05)$ & $0.35(0.05)$ \\
\hline GENO > 0.01 & 536668 & $0.23(0.03)$ & $0.23(0.05)$ & $0.35(0.05)$ \\
\hline GENO > 0.005 & 525248 & $0.22(0.03)$ & $0.22(0.05)$ & $0.35(0.05)$ \\
\hline GENO > 0.001 & 410973 & $0.21(0.03)$ & $0.21(0.05)$ & $0.35(0.05)$ \\
\hline
\end{tabular}

aEstimated genetic variance on the observed scale with SE.

${ }^{\mathrm{b}}$ Estimated genetics variance and SE on observed scale adjusted for LD after Yang et al. ${ }^{19}$

Estimated genetic variance and SE transformed to the liability scale after Dempster and Lerner. ${ }^{21}$

'Dinor allele frequency.

eMaximum per-SNP missing rate. 


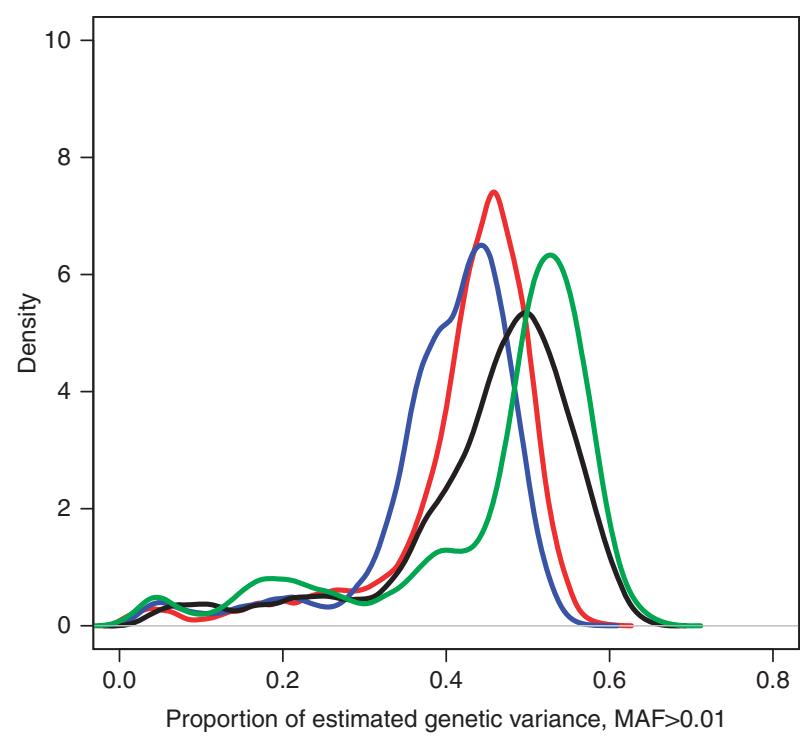

Figure 1 Proportion of estimated variance for MAF $>0.01$ with maximum per-SNP missing rate: red $=0.001 ; \quad$ blue $=0.005 ; \quad$ black $=0.01$; green $=0.05$.

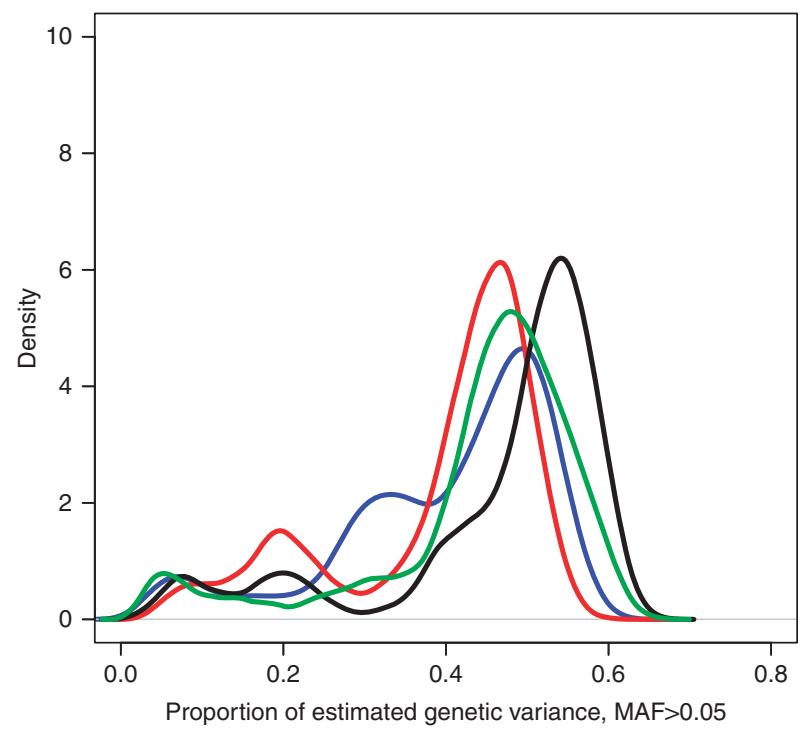

Figure 2 Proportion of estimated variance for MAF $>0.05$ with maximum per-SNP missing rate: red $=0.001 ; \quad$ blue $=0.005$; black $=0.01$; green $=0.05$.

environment would result in changes of the prevalence. Cutting the used prevalence in half to $\sim 0.5$ in 1000 or doubling it to $\sim 2$ in 1000 did not have any influence on the estimates of the common variance at any threshold shown in Table 1, but for a prevalence of $\sim 0.5$ in 1000, the transformed estimate dropped to 0.33 (SE: 0.04), whereas it increased to 0.40 (SE: 0.05) for a prevalence of $\sim 2$ in 1000 .

According to Wray et $a l^{33}$ we could interpret our result of the common genetic variance explained by SNPs on the liability scale to mean that we must expect many genetic variants underlying the disease. As the risks of common variants are too small to be used individually as risk predictors, the overall transformed estimate of 0.35
Table 3 Genetic variances explained by all SNPs by fitting first 2, 4 and 10 principal components (PCs) as covariates in the REML analysis

\begin{tabular}{llll}
\hline Threshold & $\begin{array}{c}\text { First 2 } P C S^{\mathrm{a}} \\
\text { estimate }(S E)^{\mathrm{b}}\end{array}$ & $\begin{array}{c}\text { First 4 PCS } \\
\text { estimate }(S E)^{\mathrm{b}}\end{array}$ & $\begin{array}{c}\text { First 10 PCs } \\
\text { estimate }(S E)^{\mathrm{b}}\end{array}$ \\
\hline MAF $^{\mathrm{c}} 0.01$ & & & \\
GENO $^{\mathrm{d}}>0.05$ & $0.24(0.04)$ & $0.23(0.04)$ & $0.22(0.04)$ \\
GENO $>0.01$ & $0.24(0.04)$ & $0.23(0.04)$ & $0.22(0.04)$ \\
GENO $>0.005$ & $0.24(0.04)$ & $0.23(0.04)$ & $0.21(0.04)$ \\
GENO $>0.001$ & $0.24(0.04)$ & $0.23(0.04)$ & $0.21(0.04)$ \\
& & & \\
MAF $>0.05$ & & & $0.22(0.04)$ \\
GENO $>0.05$ & $0.24(0.04)$ & $0.23(0.04)$ & $0.22(0.04)$ \\
GENO >0.01 & $0.24(0.04)$ & $0.23(0.04)$ & $0.22(0.04)$ \\
GENO >0.005 & $0.24(0.04)$ & $0.23(0.04)$ & $0.22(0.04)$ \\
GENO >0.001 & $0.24(0.04)$ & $0.23(0.04)$ & \\
\hline
\end{tabular}

aPrincipal components.

bEstimated genetic variance on the observed scale with SE.

cMaximum per-SNP missing rate.

${ }^{d}$ Minor allele frequency.

(Table 1) can be translated to a sibling relative risk of 5.58 being associated with common genetic variation. ${ }^{33}$

\section{Heritability estimates based on population data}

Figure 3 shows a trace plot of the heritability values along the iterations. There is no trend in the trace and values spread widely with a reasonable parameter space. The plot clearly illustrates good mixing, and a Gibbs sampler that 'converges' fast. The right side of Figure 3 shows the posterior density of the heritability estimate as a result of the model described earlier applied to the data set. Averaged across the 10000 samples, posterior means of the heritability were 0.40 on the liability scale with a corresponding $95 \%$ highest posterior density region (HPD95) ranging from 0.17 to 0.58 . The heritability on the observed scale is $z^{2} / K(1-K)$ times the heritability on the underlying normally distributed liability scale that is then 0.24 . According to the convergence criteria, the effective sample size for estimating the mean derived from our data set was $\sim 7186$, representing a sufficient number, given the fact that we had 10000 samples from the Gibbs sampler. ${ }^{31}$

\section{DISCUSSION}

Results from the genomic analysis as well as the population-based analysis show the proportion of variance explained by SNPs and heritability values for the susceptibility to $\mathrm{HL}$ in an overlapping range from 0.21 to 0.48 , whereas estimates on the liability scale are ranging from 0.35 to 0.48 only. Most of these values represent simply the proportion of the total variance that is attributable to the pure additive genetic variance. Estimates of the PVE as a result of the probit-based approach ranged from 0.39 to 0.48 , and were somehow higher compared with estimates of the genetic variance explained by SNPs on the liability scale shown in Table 1 . This can be explained by a slightly different concept of the PVE compared with the approaches of Yang et $a l^{19}$ and Speed et $a l^{20}$ : PVE reflects the optimal predictive accuracy achieved for a linear combination of the measured genetic variants, whereas heritability reflects the accuracy that one can achieve by using all genetic variants. ${ }^{23}$ Simulations of Guan and Stephens ${ }^{23}$ have proven that the uncertainty in PVE is greater with a larger number of SNPs, presumably because of the increased difficulty in 
Table 4 Analysis without chromosome 6 or chromosome 6 only

\begin{tabular}{|c|c|c|c|c|}
\hline Threshold & No. of SNPS & Estimate $(S E)^{a}$ & Adjusted $(S E)^{b}$ & Transformed (SE) \\
\hline \multicolumn{5}{|c|}{ Analysis without chromosome 6} \\
\hline \multicolumn{5}{|c|}{ MAF $>0.01$} \\
\hline GENO >0.05 & 539581 & $0.19(0.03)$ & $0.20(0.03)$ & $0.35(0.05)$ \\
\hline GENO >0.01 & 536307 & $0.19(0.03)$ & $0.19(0.03)$ & $0.35(0.05)$ \\
\hline GENO $>0.005$ & 525240 & $0.19(0.03)$ & $0.19(0.03)$ & $0.35(0.05)$ \\
\hline GENO >0.001 & 410698 & $0.19(0.03)$ & $0.19(0.03)$ & $0.35(0.05)$ \\
\hline \multicolumn{5}{|l|}{ MAF $>0.05$} \\
\hline GENO >0.05 & 500018 & $0.19(0.03)$ & $0.19(0.03)$ & $0.35(0.05)$ \\
\hline GENO >0.01 & 497100 & $0.19(0.03)$ & $0.19(0.03)$ & $0.35(0.05)$ \\
\hline GENO >0.005 & 486988 & $0.19(0.03)$ & $0.18(0.03)$ & $0.35(0.05)$ \\
\hline GENO $>0.001$ & 381802 & $0.19(0.03)$ & $0.18(0.03)$ & $0.35(0.05)$ \\
\hline \multicolumn{5}{|c|}{ Analysis of chromosome 6 only } \\
\hline \multicolumn{5}{|c|}{$\mathrm{MAF}^{\mathrm{d}}>0.01$} \\
\hline $\mathrm{GENO}^{\mathrm{e}}>0.05$ & 43752 & $0.05(0.01)$ & $0.04(0.01)$ & $0.10(0.01)$ \\
\hline GENO >0.01 & 43138 & $0.05(0.01)$ & $0.04(0.01)$ & $0.10(0.01)$ \\
\hline GENO $>0.005$ & 41591 & $0.05(0.01)$ & $0.04(0.01)$ & $0.10(0.01)$ \\
\hline GENO >0.001 & 31627 & $0.05(0.01)$ & $0.04(0.01)$ & $0.10(0.01)$ \\
\hline \multicolumn{5}{|l|}{$\mathrm{MAF}>0.05$} \\
\hline GENO >0.05 & 40210 & $0.04(0.01)$ & $0.04(0.01)$ & $0.10(0.01)$ \\
\hline GENO >0.01 & 39568 & $0.04(0.01)$ & $0.04(0.01)$ & $0.10(0.01)$ \\
\hline GENO $>0.005$ & 38260 & $0.04(0.01)$ & $0.04(0.01)$ & $0.10(0.01)$ \\
\hline GENO $>0.001$ & 29171 & $0.04(0.01)$ & $0.03(0.01)$ & $0.10(0.01)$ \\
\hline
\end{tabular}

aEstimated genetic variance with SE on the observed scale.

${ }^{b}$ Estimated genetic variance with SE on observed scale adjusted for LD after Yang et al 19

'Estimated genetic variance with SE transformed to the liability scale after Dempster and Lerner. ${ }^{21}$

dMinor allele frequency.

eMaximum per-SNP missing rate.

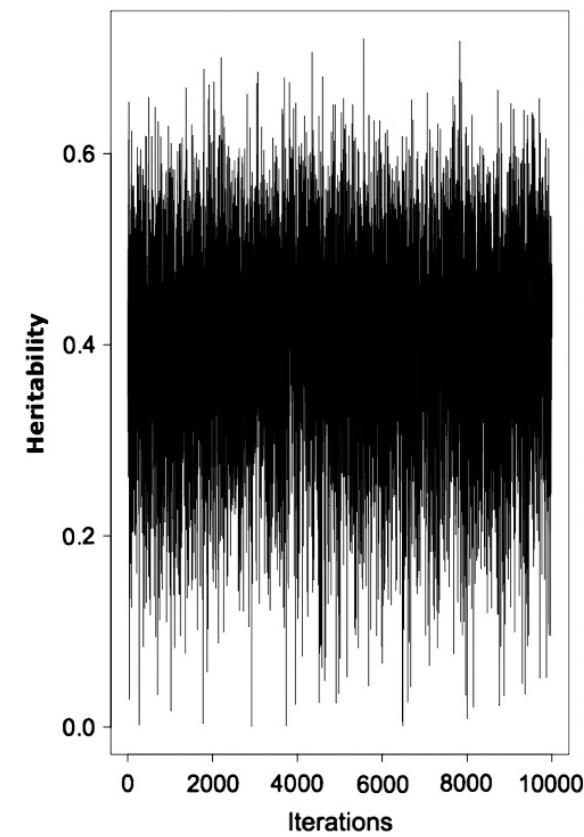

Figure 3 Trace (left) and posterior density (right) of heritability estimate.

reliably identifying relevant variants. When data contain no SNPs with strong individual effects, it remains difficult to rule out the possibility that many SNPs may have very small effects that combine to produce an appreciable PVE. ${ }^{23}$ This uncertainty is also represented by the rather large confidence intervals in the right column of Table 2. It will

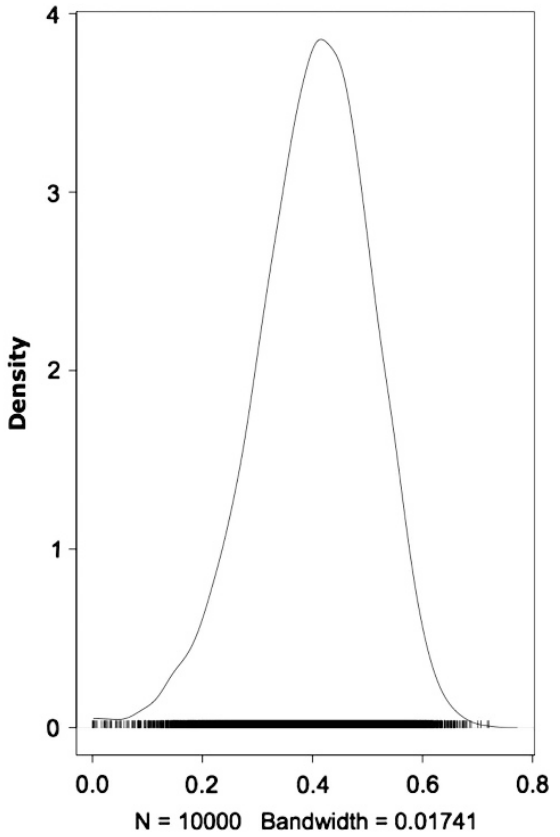

be even greater when the true PVE is smaller. ${ }^{23}$ Nonetheless, the range of the posterior on PVE nicely spans the estimates provided by the other methods equally to both sides, proving the ability of the PVE method to quantify uncertainty in multivariate problems by assessing the full joint posterior distribution of the model parameters compared 
with the current simplistic 'one SNP at a time' testing paradigm, ${ }^{34,35}$ because analyzing all SNPs simultaneously will detect more of the genetic variation because of the identification of multiple causal variants. ${ }^{36}$

In contrast to the results of Enciso-Mora et al, ${ }^{37}$ our estimates of the variance are almost constant across the different numbers of SNPs and do not decline after a more stringent exclusion of missing genotypes. This is in agreement with the results by Yang et al ${ }^{19}$ and Lee et al. ${ }^{38}$ Thus, our QC has been stringent enough beforehand.

Inflation of the estimated variances explained by SNPs due to population stratification has been investigated by genomic partitioning. Results did not provide any evidence of stratification. Estimates of the two parts of the genome added up to the corresponding estimates on the full set (right column in Table 1). Our stringent QC helped to keep the genomic control inflation factor low. ${ }^{6}$

A cause for concern in estimating the common genetic variance explained by SNPs is created by LD that can lead to large biases. Contributions to heritability estimates from causal variants might be overestimated because of regions with strong LD or underestimated in regions with low LD. ${ }^{20} \mathrm{We}$ also followed the proposal of Speed et al ${ }^{20}$ to analyze our data, but we did not detect any perceptible deviations in our estimates compared with the method of Yang et al. ${ }^{19}$ It seems like any underestimation of contributions to the heritability in low-LD regions is balanced by overestimation elsewhere as shown by Speed et al. ${ }^{20}$

Trends in cancer prevalence reveal the dynamics of cancers in the population. To test the influence of changes on the estimates of the common genetic variance explained by SNPs, we have halved and doubled the prevalence. Differences to the original prevalence could only be seen in the transformed variance, but standard estimates stayed constant. Thus, variation in the transformed estimates may reflect changes in the environment over time.

The estimates of the common genetic variance explained by SNPs are based on $\sim 410000$ to almost 600000 SNPs. A significant effect on $\mathrm{HL}$ is harbored in the MHC region on chromosome 6.,39 After excluding SNPs mapped to the MHC region (6p21, at 28-33 Mb), Enciso-Mora et $a l^{39}$ remained only with a limited number of loci influencing the risk of HL. Nonetheless, Enciso-Mora et $a^{39}$ and Frampton et al ${ }^{6}$ were able to identify suggestive associations on another eight autosomes. After we excluded chromosome 6 from our analysis, the estimates dropped by $\sim 20 \%$. A rather high proportion of the variance is thus explained by chromosome 6 only as shown in Table 4, but still a descent proportion of variance is explained by the remaining autosomes. We therefore conclude that many additional loci may contribute to the susceptibility of HL.

The link function for binary data most widely used is the probit link, also known as the threshold model. ${ }^{40}$ Modeling a random variable by using the probit function assumes that the latent variable (liability) possesses a standard normal distribution. The major advantage is that the liability is treated as a polygenic trait that is determined by many genes with small effects, and therefore heritability of liability is independent of the disease prevalence and can be directly compared. ${ }^{40}$

Our heritability estimates on the liability scale based on the Swedish population are larger than estimates by Shugart et al, ${ }^{8}$ who calculated heritability for HL to be $28.4 \%$. A reason for their lower estimate is a bias in Falconer's method ${ }^{8}$ that is a result of common familiar environmental factors and ascertainment. ${ }^{41}$ Generally, by using the extensive pedigree with its whole range of relationships in the population, the so-called animal threshold model provides the most accurate approximation of the heritability. ${ }^{42}$ Our estimates are larger, but still conservative, because the applied threshold model only estimated the contributions of genes that act additively: the effects of genes with nonadditive effects, such as dominance or epistasis, will not contribute to the heritability estimates reported here. And even though statistical models are available for the estimation of nonadditive genetic variance, much larger sets of suitably structured data would be required to obtain reliable estimates.

Heritability estimates on the liability scale are slightly larger for the population-based data than estimates of the common genetic variance explained by SNPs ( 0.40 compared with 0.35 for the GCTA approach), but they were in a very similar range to PVE (Table 2) that gave larger estimates because of reasons explained above. Nonetheless, heritability estimated from pedigree data is not the same as the proportion of phenotypic variation explained by all SNPs because the former includes the contribution of all causal variants, but the latter only includes the contribution of causal variants that are in LD with the genotyped SNPs. Thus, we also face the problem of missing heritability known for analysis of GWAS data. ${ }^{11}$ Our population-based estimates and the estimates from SNP data still do not differ too much as estimates for other traits. ${ }^{16}$ We therefore conclude that our genotypic data is a well-formed sample to draw sufficient conclusion about the genetic determination of the disease. Even though estimates of the common genetic variance explained by SNPs and the populationbased heritability are similar, we must point out that many reasons for missing heritability have been widely accepted in the scientific community. This knowledge is supported by our findings of different estimates of the genetic variance explained by SNPs on chromosome 6 compared with other chromosomes. Genes detected on chromosome 6 in earlier studies do not account for much of the heritability of HL in our study. ${ }^{7,39}$ The susceptibility to HL is rather a combined effect of multiple genes on several chromosomes than that of a few disease genes on a single chromosome.

The common genetic variance explained by SNPs and the heritability on the liability scale of HL show that a reasonable proportion of the variation observed in both the German and the Swedish population is caused by variation in genotypes, but it also indicates that the environment is still the principal causative role in HL, and susceptibility genes described so far for HL are likely to explain only part of the genetic effects.

An important fact is the interpretation of the sibling relative risk that has be derived through the incidence of the disease and the estimates of common variation explained by SNPs. Based on our results, siblings of people with $\mathrm{HL}$ are $\sim 5.6$ times more likely to develop the disease than others. These results are in agreement with studies showing up to a sevenfold increased risk in people with a parent or sibling diagnosed with HL. ${ }^{43}$ It therefore seems to be clear that besides the environment, genetic factors have strong influence on the etiology of HL.

In conclusion, there is genetic variation for the susceptibility to HL. Heritability based on the population data is somehow larger than for the genomic data showing the possibility of some missing heritability in the GWAS data. Besides that, there is still major evidence for multiple loci causing HL on chromosomes other than chromosome 6.

\section{CONFLICT OF INTEREST}

The authors declare no conflict of interest.

\section{ACKNOWLEDGEMENTS}

We are grateful for the technical support on the program package DMU by Per Madsen and Guosheng Su. 
1 Bose S, Ganesan C, Pant M, Lai C, Tabbara IA: Lymphocyte-predominant Hodgkin disease: a comprehensive overview. Am J Clin Oncol 2013; 36: 91-96.

2 Engert A, Horning SJ: Hodgkin Lymphoma. A Comprehensive Update on Diagnostics and Clinics: Hematologic malignancies. Heidelberg; New York: Springer-Verlag, 2011 pp 1, online resource (ix, 381 p)

3 Parkin DM: 11. Cancers attributable to infection in the UK in 2010. Br J Cancer 2011; 105(Suppl 2): S49-S56.

4 Anderson LA, Gadalla S, Morton LM et al: Population-based study of autoimmune conditions and the risk of specific lymphoid malignancies. Int J Cancer 2009; 125: 398-405.

5 Hemminki K, Czene K: Attributable risks of familial cancer from the Family-Cancer Database. Cancer Epidemiol Biomarkers Prev 2002; 11: 1638-1644.

6 Frampton M, da Silva Filho MI, Broderick P et al: Variation at 3p24.1 and 6q23.3 influences the risk of Hodgkin's lymphoma. Nat Commun 2013; 4: 2549.

7 Urayama KY, Jarrett RF, Hjalgrim H et al: Genome-wide association study of classical Hodgkin lymphoma and Epstein-Barr virus status-defined subgroups. J Nat/ Cancer Inst 2012; 104: 240-253.

8 Shugart $Y Y$, Hemminki $K$, Vaittinen $P$, Kingman A, Dong C: A genetic study of Hodgkin's lymphoma: an estimate of heritability and anticipation based on the familial cancer database in Sweden. Hum Genet 2000; 106: 553-556.

9 Eichler EE, Flint J, Gibson G et al: Missing heritability and strategies for finding the underlying causes of complex disease. Nat Rev Genet 2010; 11: 446-450.

10 Wray NR, Yang J, Hayes BJ, Price AL, Goddard ME, Visscher PM: Pitfalls of predicting complex traits from SNPs. Nat Rev Genet 2013; 14: 507-515.

11 Bloom JS, Ehrenreich IM, Loo WT, Lite TL, Kruglyak L: Finding the sources of missing heritability in a yeast cross. Nature 2013; 494: 234-237.

12 Wilson AJ: Why h2 does not always equal VA/VP? J Evol Biol 2008; 21: 647-650.

13 Hill WG: Understanding and using quantitative genetic variation. Philos Trans $R$ Soc Lond B Biol Sci 2010; 365: 73-85.

14 Vinkhuyzen AA, Wray NR, Yang J, Goddard ME, Visscher PM: Estimation and partition of heritability in human populations using whole-genome analysis methods. Annu Rev Genet 2013; 47: 75-95.

15 Falconer DS: Introduction to Quantitative Genetics, 3rd edn. Burnt Mill, Harlow, Essex, England New York: Longman, Wiley, 1989.

16 Lee SH, Wray NR, Goddard ME, Visscher PM: Estimating missing heritability for disease from genome-wide association studies. Am J Hum Genet 2011; 88: 294-305.

17 Schmermund A, Mohlenkamp S, Stang A et al: Assessment of clinically silent atherosclerotic disease and established and novel risk factors for predicting myocardia infarction and cardiac death in healthy middle-aged subjects: rationale and design of the Heinz Nixdorf RECALL Study. Risk Factors, Evaluation of Coronary Calcium and Lifestyle. Am Heart J 2002; 144: 212-218.

18 Purcell S, Neale B, Todd-Brown K et al: PLINK: a tool set for whole-genome association and population-based linkage analyses. Am J Hum Genet 2007; 81: 559-575.

19 Yang J, Benyamin B, McEvoy BP et al: Common SNPs explain a large proportion of the heritability for human height. Nat Genet 2010; 42: 565-569.

20 Speed D, Hemani G, Johnson Michael R, Balding David J: Improved Heritability Estimation from Genome-wide SNPs. Am J Hum Genet 2012; 91: 1011-1021.

21 Dempster ER, Lerner IM: Heritability of threshold characters. Genetics 1950; 35 212-236.

22 Yang J, Lee SH, Goddard ME, Visscher PM: GCTA: a tool for genome-wide complex trait analysis. Am J Hum Genet 2011; 88: 76-82.
23 Guan Y, Stephens M: Bayesian variable selection regression for genome-wide association studies and other large-scale problems. Ann Appl Stat 2011; 5 1780-1815.

24 Hemminki K, Ji J, Brandt A, Mousavi SM, Sundquist J: The Swedish Family-Cancer Database 2009: prospects for histology-specific and immigrant studies. Int J Cancer 2010; 126: 2259-2267.

25 R Core Team: R: A Language and Environment for Statistical Computing. Vienna, Austria: R Foundation for Statistical Computing, 2013, ISBN 3-900051-07-0. http:// www.R-project.org/.

26 Madsen P: User's Guide to DmuTrace: A Package for Preparing Data Sets, Version 2. Aarhus, UK: University of Aarhus, Faculty of Agricultural Sciences, Department of Animal Breeding and Genetics, 2012.

27 Sorensen D, Gianola D: Likelihood, Bayesian and MCMC Methods in Quantitative Genetics. New York: Springer-Verlag, 2002.

28 Odegard J, Meuwissen TH, Heringstad B, Madsen P: A simple algorithm to estimate genetic variance in an animal threshold model using Bayesian inference. Genet Sel Evol 2010; 42: 29.

29 Lynch M, Walsh B: Genetics and Analysis of Quantitative Traits. Sunderland, MA: Sinauer, 1998

30 Madsen P, Jensen J: DMU: A User's Guide. A Package for Analyzing Multivariate Mixed Models, Version 6, release 4.7. Aarhus, UK: University of Aarhus, Faculty of Agricultural Sciences, Department of Animal Breeding and Genetics, 2007.

31 Hadfield JD: MCMC methods for multi-response generalized linear mixed models: the MCMCgImm R Package. J Stat Softw 2010; 33: 1-22.

32 Yang J, Manolio TA, Pasquale LR et al: Genome partitioning of genetic variation for complex traits using common SNPs. Nat Genet 2011; 43: 519-525.

33 Wray NR, Yang J, Goddard ME, Visscher PM: The genetic interpretation of area unde the ROC curve in genomic profiling. PLoS Genet 2010; 6: e1000864.

34 Gelman A: Bayesian Data Analysis, 2nd edn. Boca Raton, FL: Chapman \& Hall/CRC 2004.

35 Stephens M, Balding DJ: Bayesian statistical methods for genetic association studies. Nat Rev Genet 2009; 10: 681-690

36 Ovaskainen O, Cano JM, Merila J: A Bayesian framework for comparative quantitative genetics. Proc Biol Sci R Soc 2008; 275: 669-678.

37 Enciso-Mora V, Hosking FJ, Sheridan E et al: Common genetic variation contributes significantly to the risk of childhood B-cell precursor acute lymphoblastic leukemia. Leukemia 2012; 26: 2212-2215.

38 Lee SH, DeCandia TR, Ripke S et al: Estimating the proportion of variation in susceptibility to schizophrenia captured by common SNPs. Nat Genet 2012; 44 247-250.

39 Enciso-Mora V, Broderick P, Ma Y et al: A genome-wide association study of Hodgkin's lymphoma identifies new susceptibility loci at 2p16.1 (REL), 8q24.21 and 10p14 (GATA3). Nat Genet 2010; 42: 1126-1130.

40 Gianola D, Foulley J: Sire evaluation for ordered categorical data with a threshold model. Genet Sel Evol 1983; 15: 201-224.

41 Tenesa A, Haley CS: The heritability of human disease: estimation, uses and abuses. Nat Rev Genet 2013; 14: 139-149.

42 Benchek P, Morris NJ: How meaningful are heritability estimates of liability? Hum Genet 2013; 132: 1351-1360.

43 Goldin LR, Pfeiffer RM, Gridley G et al: Familial aggregation of Hodgkin lymphoma and related tumors. Cancer 2004; 100: 1902-1908. 